\title{
Adaptive Optical Phase Estimation Using Time-Symmetric Quantum Smoothing
}

\author{
T. A. Wheatley, ${ }^{1,2,3}$ D. W. Berry ${ }^{4}$ H. Yonezawa, ${ }^{3}$ D. Nakane,${ }^{3}$ H. Arao, ${ }^{3}$ D. T. Pope ${ }^{5}$ \\ T. C. Ralph, ${ }^{1,6, *}$ H. M. Wiseman, ${ }^{1,7, \dagger}$ A. Furusawa, ${ }^{3, \ddagger}$ and E. H. Huntington ${ }^{1,2, \S}$ \\ ${ }^{1}$ Centre for Quantum Computer Technology, Australian Research Council \\ ${ }^{2}$ School of Engineering and Information Technology, University College, \\ The University of New South Wales, Canberra 2600, ACT, Australia \\ ${ }^{3}$ Department of Applied Physics and Quantum Phase Electronics Center, School of Engineering, \\ The University of Tokyo, 7-3-1 Hongo, Bunkyo-ku, Tokyo 113-8656, Japan \\ ${ }^{4}$ Institute for Quantum Computing, University of Waterloo, Waterloo, ON, Canada \\ ${ }^{5}$ Perimeter Institute for Theoretical Physics, 31 Caroline Street N, Waterloo, ON N2L 2Y5, Canada \\ ${ }^{6}$ Department of Physics, University of Queensland, Brisbane 4072, QLD, Australia \\ ${ }^{7}$ Centre for Quantum Dynamics, Griffith University, Brisbane 4111, QLD, Australia
}

\begin{abstract}
Quantum parameter estimation has many applications, from gravitational wave detection to quantum key distribution. The most commonly used technique for this type of estimation is quantum filtering, using only past observations. We present the first experimental demonstration of quantum smoothing, a time-symmetric technique that uses past and future observations, for quantum parameter estimation. We consider both adaptive and non-adaptive quantum smoothing, and show that both are better than their filtered counterparts. For the problem of estimating a stochastically varying phase shift on a coherent beam, our theory predicts that adaptive quantum smoothing (the best scheme) gives an estimate with a mean-square error up to $2 \sqrt{2}$ times smaller than non-adaptive filtering (the standard quantum limit). The experimentally measured improvement is $2.24 \pm 0.14$.
\end{abstract}

PACS numbers: 42.50.Dv, 42.50.Xa, 03.65.Ta, 03.67.-a, 06.90.+v

Quantum parameter estimation (QPE) is the problem of estimating an unknown classical parameter (or process) which plays a role in the preparation (or dynamics) of a quantum system $[1,2]$, and is central to many fields including gravitational wave interferometry [5], quantum computing [3], and quantum key distribution [4]. The fundamental limit to the precision of the estimate in QPE is set by quantum mechanics $[1,2]$. Thus one of the key issues in QPE is the development of practical methodologies which allow measurements to approach or exceed the standard quantum limit (SQL) for a given measurement coupling [6-12]. Because of its wide-ranging technological relevance, the prime example of QPE is estimating an optical phase shift [13-20].

Apart from some theoretical papers [19, 20], work in this area of QPE has concentrated upon the problem of estimating a fixed, but unknown phase shift, which can be thought of as preparing the quantum state with an average phase equal to this parameter. It was shown theoretically [15] that for this problem adaptive homodyne measurements coupled with an optimal estimation filter can yield an estimate with mean-square error smaller than the standard quantum limit (as set by perfect heterodyne detection). This was demonstrated experimentally in Ref. [16] using very weak coherent states (for which the factor of improvement is at most 2). More recent theory and experiment have shown that interferometric measurements with photon counting can also be improved using adaptive techniques [17, 18].

A far richer, and in many cases more experimentally relevant, problem of quantum phase estimation arises when the phase evolves dynamically under the influence of an unknown classical stochastic process [19, 20]. The general problem of estimating a classical process dynamically coupled to a quantum system under continuous measurement has recently been considered by Tsang [21], who introduced three main categories of quantum estimation: prediction or filtering, smoothing, and retrodiction. Of those, prediction or filtering is a causal estimation technique that can be used in real-time applications [24]. Smoothing and retrodiction are acausal and so cannot be used in real time, but they can be used for off-line data processing or with a delay corresponding to the estimation time. Smoothing, in which the signal is inferred at a point in time based on data taken both before and after that time, is the only time-symmetric estimation technique. As a consequence, it can be more precise than the time asymmetric techniques of filtering or retrodiction $[20,21]$. Such a result is very significant for quantum sensing applications where it is more important to have precise rather than real-time estimates.

Here we present the first experimental demonstration of QPE using quantum smoothing. Specifically, we consider estimation of the phase of a continuous optical field, generalizing the theory of Ref. [19] to a more general classical phase noise process (rather than pure diffusion), and to smoothing (rather than filtering). According to our theory, adaptive measurements and smoothing both offer improvements over the alternative (non-adaptive and filtering respectively). Moreover, using both together offers the maximum improvement, with a mean-square phase error smaller than the standard (non-adaptive, filtered) quantum limit by a factor of up to $2 \sqrt{2}$ in theory for pure phase diffusion. We verify these predicted improvements 
experimentally, for the first time in every case, and find a maximum improvement by a factor of $2.24 \pm 0.14$ over the SQL.

Fig. 1 illustrates the system under consideration. The goal in this quantum sensing problem is to form the optimal estimate $\Theta(t)$ of the system phase $\varphi(t)$ of a weak coherent state in the presence of noise in the measurement and classical noise in the system phase. The precision of the estimate is quantified by the mean-square error between the estimate and the actual phase such that $\sigma^{2} \equiv\left\langle[\Theta(t)-\varphi(t)]^{2}\right\rangle$.

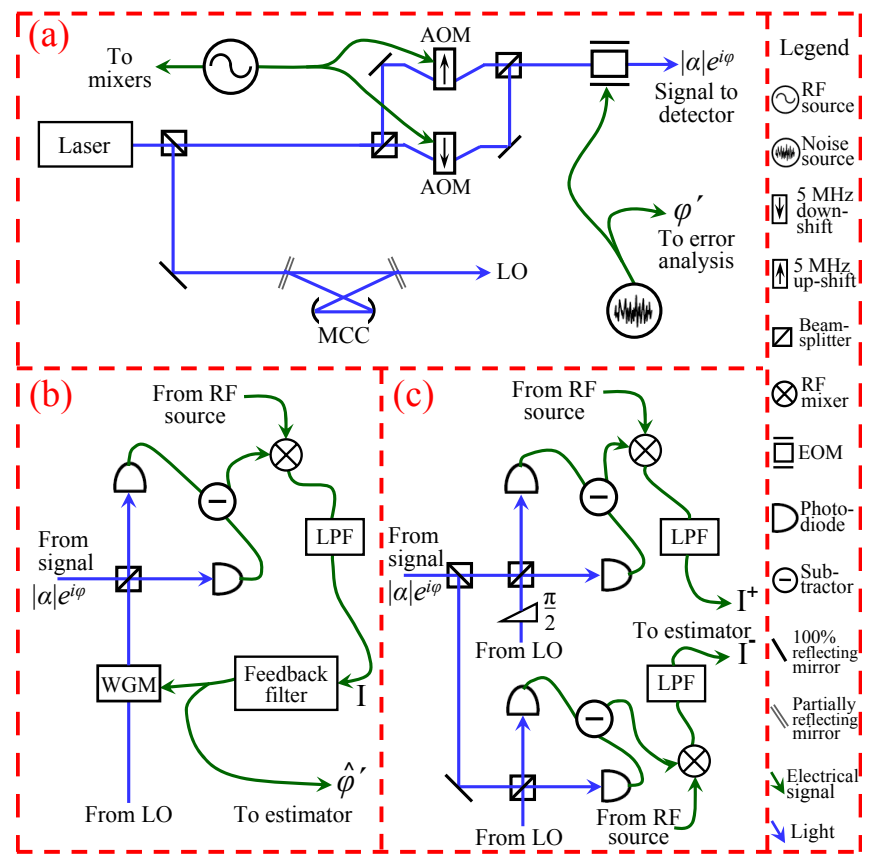

FIG. 1: Schematic diagrams showing: (a) signal and local oscillator generation; (b) adaptive phase estimation; (c) dual homodyne phase estimation. $\mathrm{LO}=$ local oscillator; $\mathrm{RF}=$ radio-frequency; $\mathrm{EOM}=$ electro-optic modulator; WGM = waveguide modulator; $\mathrm{LPF}=$ low-pass filter; $\mathrm{MCC}=$ modecleaning cavity; $\mathrm{AOM}=$ acousto-optic modulator.

Unlike previous adaptive phase estimation experiments $[16,18]$, we compare the phase estimate to the actual system phase in order to directly measure the error in estimation. This is achieved by deliberately imposing classical phase noise via an EOM, as indicated in Fig. 1(a). A titanium:sapphire laser operating at $860 \mathrm{~nm}$ is used to drive the experiment. The arrangement of AOMs shown in Fig. 1(a) is used to generate a pair of phase modulation sidebands at $\pm 5 \mathrm{MHz}$ such that it is equivalent to a weak coherent state with a photon flux $\mathcal{N}=|\alpha|^{2}$ of order $10^{6}$ photons per second.

The phase noise is applied using a EOM driven by an Ornstein-Uhlenbeck (OU) [22] noise source. The phase variation is

$$
d \varphi(t)=-\lambda \varphi(t) d t+\sqrt{\kappa} d V(t)
$$

where $d V$ is a Wiener increment and $\kappa$ is the inverse coherence time. For this experiment $\kappa$ is of order $10^{4} \mathrm{rad} / \mathrm{s}$, so there are about 100 photons per coherence time. We record the imposed voltage at the monitor port of the high voltage amplifier ( $\varphi^{\prime}$ in Fig.1). The phase deviation arising from that voltage is calibrated via the half-wave voltage of the EOM and used as our measure of the true system phase $\varphi$.

An arbitrary quadrature of the field of interest can be measured with a balanced homodyne detector, in which the field of interest is interfered with a $1.5 \mathrm{~mW}$ local oscillator on a beamsplitter. Both outputs of the beamsplitter are detected and the resulting measurements are subtracted to form the homodyne photocurrent $I(t)[23]$. The detection efficiency (including homodyne fringe visibility of $97 \%$, detector quantum efficiency of $98 \%$, and optical transmission of $97 \%$ ) was in excess of $89 \%$ in all measurements and the electronic noise floor was $11 \mathrm{~dB}$ below the shot noise of the measurements. In all cases, the homodyne detector is DC-locked to ensure that the deliberately imposed OU noise dominates the uncertainty in the phase.

The adaptive phase estimation system is illustrated in Fig. 1(b). The output of the homodyne detector is electronically demodulated to give $I(t)$ which is then fed into the feedback filter. This yields a voltage $\hat{\varphi}^{\prime}$, which is stored for later data analysis and also fed back so as to imprint a phase $\hat{\varphi} \propto \hat{\varphi}^{\prime}$ on the optical LO using a WGM. That is, the intermediate phase estimate $\hat{\varphi}$ is the phase of the measured quadrature.

Because $\varphi \approx \hat{\varphi}$, we can use a linearized approximation for the homodyne photocurrent:

$$
I(t) d t=2|\alpha|[\varphi(t)-\hat{\varphi}(t)] d t+d W(t),
$$

where $d W(t)$ is Wiener noise arising from the quantum vacuum fluctuations. We define the instantaneous estimate $\theta(t)$ to be the best estimate of $\varphi(t)$ which can be made using only the data taken in the time interval $[t, t+d t)$

$$
\begin{aligned}
\theta(t) & :=\hat{\varphi}(t)+I(t) / 2|\alpha| \\
& =\varphi(t)+\frac{d W(t)}{d t} \frac{1}{2 \sqrt{\mathcal{N}}} .
\end{aligned}
$$

To obtain an intermediate estimate $\hat{\varphi}$ with a finite amount of noise it is necessary to time-average the instantaneous estimate. This can be achieved by using a simple integrator on $I(t)$ [19], but for practical reasons we use a linear low-pass filter:

$$
\hat{\varphi}(t)=\int_{-\infty}^{t} \beta e^{\omega_{0}(s-t)} I(s) / 2 \sqrt{\mathcal{N}} d s .
$$

We work in the range where the ratio of cut-off frequency $\left(\omega_{0}=1.5 \times 10^{5} \mathrm{~s}^{-1}\right)$ to feedback gain $(\beta)$ varies from 0.02 to 0.09 , so we can assume that $\omega_{0} \ll \beta$. In this 
limit, it is easy to verify from the above equations that the intermediate estimate reduces to the filter used in Ref. [19]:

$$
\hat{\varphi}(t)=\int_{-\infty}^{t} \beta e^{\beta(s-t)} \theta(s) d s .
$$

The intermediate estimate $\hat{\varphi}(t)$ of $\varphi(t)$ is always a filtered estimate, because it is used in a causal feedback loop. The theory of Ref. [19] also used filtering to obtain the final phase estimate for $\varphi(t)$. That is, the final estimate for time $t$ was based only on the data obtained up to time $t$. However, it is possible to obtain a better phase estimate by smoothing: using the data after time $t$ also. Here we can assume that data for an infinite period of time before and after $t$ can be used, because the experimental data run time of $10^{-2} \mathrm{~s}$ is long compared to the averaging time, which is $\lesssim 10^{-4} \mathrm{~s}$.

Let us denote by $\Theta_{-}(t)$ and $\Theta_{+}(t)$ the phase estimates obtained from data obtained before and after time $t$, respectively. Following Ref. [19], we consider estimates that are weighted averages of the instantaneous estimates:

$$
\Theta_{ \pm}(t)= \pm \chi_{ \pm} \int_{t}^{ \pm \infty} \theta(s) e^{\mp \chi_{ \pm}(s-t)} d s
$$

The deviation of these from the actual phase is then

$$
\begin{aligned}
\Theta_{ \pm}(t)-\varphi(t)= & \pm \chi_{ \pm} \int_{t}^{ \pm \infty} e^{\mp \chi_{ \pm}(s-t)}[\varphi(s)-\varphi(t)] d s \\
& \pm \frac{\chi_{ \pm}}{2 \sqrt{N}} \int_{t}^{ \pm \infty} e^{\mp \chi_{ \pm}(s-t)} d W(s) .
\end{aligned}
$$

The forwards estimate, $\Theta_{-}(t)$ corresponds to the causal (or filtered) estimate. A weighted average of the forwards and backwards estimates can be used to construct the time-symmetric (or smoothed) estimate $\Theta(t)=$ $w_{-} \Theta_{-}(t)+w_{+} \Theta_{+}(t)$, the variance of which is:

$$
\begin{aligned}
\sigma^{2}= & w_{-}^{2}\left\langle\left[\Theta_{-}(t)-\varphi(t)\right]^{2}\right\rangle+w_{+}^{2}\left\langle\left[\Theta_{+}(t)-\varphi(t)\right]^{2}\right\rangle \\
& +2 w_{-} w_{+}\left\langle\left[\Theta_{-}(t)-\varphi(t)\right]\left[\Theta_{+}(t)-\varphi(t)\right]\right\rangle .
\end{aligned}
$$

This can be evaluated using the definition of the OU process for the system phase (1). From the time symmetry of this process, the mean-square errors in $\Theta_{+}(t)$ and $\Theta_{-}(t)$ are the same:

$$
\sigma_{ \pm}^{2}=\frac{\kappa}{2\left(\chi_{ \pm}+\lambda\right)}+\frac{\chi_{ \pm}}{8 \mathcal{N}}
$$

while the correlation between the forwards and backwards estimates is

$$
\left\langle\left[\Theta_{-}(t)-\varphi(t)\right]\left[\Theta_{+}(t)-\varphi(t)\right]\right\rangle=\frac{\kappa \lambda}{2\left(\chi_{-}+\lambda\right)\left(\chi_{+}+\lambda\right)} .
$$

By symmetry, Eq. (9) expression is minimised for $\chi_{-}=$ $\chi_{+}=\chi$ and $w_{-}=w_{+}=1 / 2$, which gives

$$
\sigma^{2}=\frac{\kappa(\chi+2 \lambda)}{4(\chi+\lambda)^{2}}+\frac{\chi}{16 \mathcal{N}}
$$

Equations (10) and (12) are simplified greatly in the limit $\xi \equiv \lambda /(2 \sqrt{\kappa \mathcal{N}}) \ll 1$, which is a good guide for our experiment where $\xi \approx 0.2$. In this limit, the optimal value of $\chi$ in Eq. (10) is $2 \sqrt{\kappa \mathcal{N}}$, giving the minimum variance $\sigma_{-}^{2}=\sqrt{\kappa / \mathcal{N}} / 2$. The relative corrections are $O\left(\xi^{2}\right)$. Changing to smoothing reduces the variance by a factor of 2 to $\sigma^{2}=\sqrt{\kappa / \mathcal{N}} / 4$.

We compare the results of the adaptive technique to the standard technique for phase estimation, dual homodyne detection, illustrated in Fig. 1(c). It incurs the same noise penalty as heterodyne detection. The dual homodyne data $\left[I^{+}\right.$and $I^{-}$in Fig. 1(c)] can be used to form an instantaneous estimate, comparable to Eq. (3), via

$$
\theta_{\mathrm{S}}(t)=\arg \left[I_{+}(t)+i I_{-}(t)\right]
$$

This can be shown to give an estimate which is effectively the same as Eq. (4), but with an additional noise penalty incurred from measuring both quadratures: $\mathcal{N}$ must be replaced by $\mathcal{N}_{\mathrm{s}}=\mathcal{N} / 2$ [19]. The mean-square errors for dual homodyne measurements are simply obtained by making this substitution in Eqs. (10) and (12). Comparing these to the adaptive results, the latter thus give a reduction in the variance by a factor of $\sqrt{2}$ for both filtering and smoothing. The SQL is set by nonadaptive filtering, and equals $\sigma_{s-}^{2}=\sqrt{\kappa / \mathcal{N}_{\mathrm{s}}} / 2$.

The measured and predicted mean-square errors for the four different estimation techniques are shown in Fig. 2. The values of $\kappa$ and $\lambda$ are determined from the calibrated measurements of the system phase $\varphi(t)$. The photon number, $\mathcal{N}=\left|\alpha^{2}\right|$, is from the measured amplitude of the coherent state relative to the quantum noise limit, while $\chi$ is varied in the experiment as indicated in Fig. 2. Each data run is $10 \mathrm{~ms}$ long, and error bars are the standard deviation of multiple data sets. We performed the adaptive estimates $\Theta_{ \pm}(t)$ not by averaging $\theta(t)$ as in Eq. (7), but rather by averaging $\hat{\varphi}(t)$. This gives more stable results, and is justified since, in the limit $\xi \ll 1$, the optimal value of $\beta$ in Eq. (5) is $\sqrt{8 \chi \mathcal{N}}[19]$. In the regime of the experiment, this $\beta$ is much greater than $\chi$, so the extra averaging in Eq. (6) is negligible.

Figure 2 shows good agreement between theory and experiment for all the estimation techniques. It demonstrates that phase estimation by quantum smoothing is significantly better than that from quantum filtering. As predicted, the improvement is nearly a factor of two at the optimum value of $\chi$ for both the adaptive and dual homodyne measurements. Figure 2 also shows the first experimental verification of the quantum theory of continuous adaptive phase estimation [19]. As predicted, adaptive phase estimation outperforms dual homodyne measurement by a factor of approximately $\sqrt{2}$. The theory curves here take into account the known imperfections (detector dark noise, homodyne efficiency and optical transmission losses). However, the horizontal line indicating the SQL $\sigma_{s-}^{2}$ is defined (as above) in terms of 


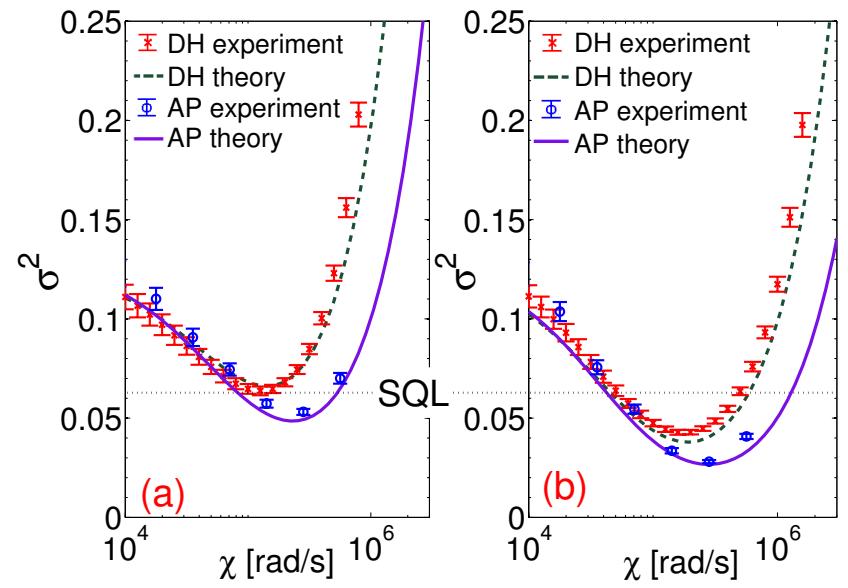

FIG. 2: The experimental and theoretical variance $\sigma^{2}$ of the four phase estimation techniques: filtered dual homodyne (DH) and adaptive phase (AP) in part (a); and smoothed $\mathrm{DH}$ and $\mathrm{AP}$ in part (b). Parameters are: $\kappa_{D H}=1.6218 \times$ $10^{4} \mathrm{rad} / \mathrm{s}, \lambda_{D H}=6.4593 \times 10^{4} \mathrm{rad} / \mathrm{s}, \mathcal{N}_{D H}=1.3235 \times 10^{6}$ $\mathrm{s}^{-1}, \kappa_{A P}=1.5868 \times 10^{4} \mathrm{rad} / \mathrm{s}, \lambda_{A P}=6.1451 \times 10^{4} \mathrm{rad} / \mathrm{s}$, $\mathcal{N}_{A P}=1.3499 \times 10^{6} \mathrm{~s}^{-1}$.

the actual photon flux, and corresponds to what would be achievable by ideal dual-homodyne filtering. Note that adaptive measurements perform better than the SQL for both types of estimator.

Figure 3 shows the optimal mean-square errors in the filtered and smoothed adaptive estimates for four different values of measured photon number, spanning an order of magnitude between $\mathcal{N} \approx 10^{6} \mathrm{~s}^{-1}$ and $\mathcal{N} \approx 10^{7} \mathrm{~s}^{-1}$. The results confirm that quantum smoothing outperforms quantum filtering over a wide range of photon numbers, as predicted by theory. As in Fig. 2, the SQL is set by ideal dual homodyne filtering, while the other theory lines are for the actual (non-ideal) experiment. As predicted, adaptive measurements out-perform the SQL.

In summary, we have demonstrated experimentally and theoretically that estimation of the phase of an optical field in the presence of classical noise using quantum smoothing is superior to the equivalent quantum filtered approach. We have also demonstrated experimentally for the first time that continuous adaptive measurements perform better than the standard quantum limit for both types of estimator. Combining quantum smoothing with adaptive measurements gives the maximum improvement over the standard (perfect non-adaptive, filtering) quantum limit. The experimental improvement of a factor of $2.24 \pm 0.14$ in the mean-square error compares well with the theoretical maximum (using phase diffused coherent states) of $2 \sqrt{2}$. These insights and techniques will be applicable to the even more interesting case of estimation using non-classical states, where the improvement can be arbitrarily large.

This work was supported financially by SCF, GIA, GCOE, PFM of MEXT, REFOST, SCOPE of MIC, the

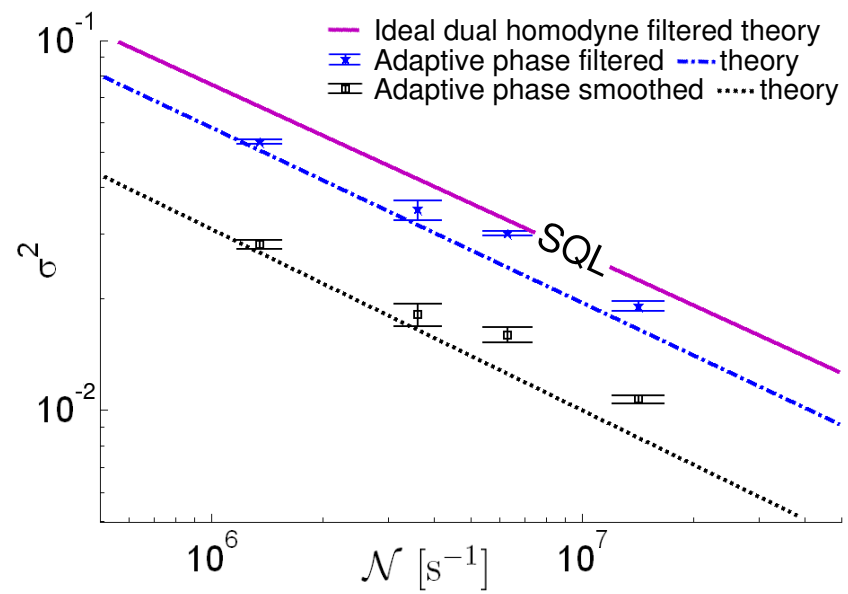

FIG. 3: The variance $\sigma^{2}$ of the adaptive phase estimation for quantum filtering and smoothing as a function of photon number $\mathcal{N}$, compared to the relevant theoretical predictions, and the theoretical predictions for nonadaptive measurements.

ARC, Industry Canada and the Province of Ontario.

* Electronic address: ralph@physics.uq.edu.au

$\dagger$ Electronic address: h.wiseman@griffith.edu.au

¥ Electronic address: akiraf@ap.t.u-tokyo.ac.jp

$\S$ Electronic address: e.huntington@adfa.edu.au

[1] V. Giovannetti, S. Lloyd, and L. Maccone, Science 306, 1330 (2004).

[2] H. M. Wiseman and G. J. Milburn, Quantum Measurement and Control, (Cambridge University Press, Cambridge, 2010)

[3] M. Hofheinz et al., Nature 459, 546, (2009).

[4] K. Inoue, E. Waks, and Y. Yamamoto. Phys. Rev. Lett. 89, 037902 (2002).

[5] K. Goda et al., Nature Physics 4, 472 (2008).

[6] J. M. Geremia, J. K. Stockton, A. C. Doherty, and H. Mabuchi, Phys. Rev. Lett. 91, 250801 (2003).

[7] C. Silberhorn, Contemporary Physics 48, 143 (2007).

[8] W. van Dam et al., Phys. Rev. Lett. 98, 090501 (2007).

[9] A. Lupascu et al., Nature Physics 3, 119 ( 2007).

[10] A. M. Brańczyk et al., Phys. Rev. A 75, 012329 (2007).

[11] U. L. Andersen, M. Sabuncu, R. Filip, and G. Leuchs, Phys. Rev. Lett. 96, 020409 (2006).

[12] R. L. Cook, P. J. Martin, and J. M. Geremia, Nature 446, 774 (2007).

[13] G. S. Summy and D. T. Pegg, Opt. Comm. 77, 75 (1990).

[14] B. C. Sanders and G. J. Milburn, Phys. Rev. Lett. 75, 2944 (1995).

[15] H. M. Wiseman, Phys. Rev. Lett. 75, 4587 (1995); H. M. Wiseman and R. B. Killip, Phys. Rev. A 56, 944 (1997); and H. M. Wiseman and R. B. Killip, Phys. Rev. A 57, 2169 (1998).

[16] M. A. Armen et al., Phys. Rev. Lett. 89, 133602 (2002).

[17] D. W. Berry and H. M. Wiseman, Phys. Rev. Lett. 85, 5098 (2000).

[18] B. L. Higgins et al., Nature 450, 393 (2007); and B. L. 
Higgins et al., New J. Phys. 11, 073023 (2009).

[19] D. W. Berry and H. M. Wiseman, Phys. Rev. A 65, 043803 (2002).

[20] M. Tsang, J. H. Shapiro, and S. Lloyd, Phys. Rev. A 79, 053843 (2009).

[21] M. Tsang, Phys. Rev. Lett. 102, 250403 (2009).

[22] C. W. Gardiner, Handbook of Stochastic Methods
(Springer, Berlin, 2004).

[23] H-A. Bachor and T. C. Ralph, A Guide to Experiments in Quantum Optics. (Wiley-VCH, Weinheim, 2004).

[24] Tsang [21] makes the distinction that prediction uses data taken prior to the time of the estimate, whereas filtering uses data taken up to the time of the estimate. 